\title{
Experiences in growing a skin radiation therapy practice
}

\begin{abstract}
The successful treatment of skin disease decreases morbidity and mortality. Radiation therapy (RT) can cure skin disease and conserves tissue, possibly delivering better quality of life post treatment and so a superior survivorship. Modern RT delivers better dose conformality and homogeneity, and more is known about the radiobiology of skin and its diseases, enabling treatment personalisation. Skin, however, can be viewed in RT departments as not a serious subspecialty, even in Australia where the incidence of skin cancer is highest. Radiation oncology leaders are needed to carve out a niche for RT amongst a crowded field of skin carers. This article is based on the cumulative experience of a group of Australian skin radiation oncologists (ROs) and details how this may be achieved. First, focus is placed on growing a high-quality service. The RO needs to understand how patient, tumour and treatment factors impact the skin RT prescription. The particular nuances around skin RT planning, including immobilisation, simulation, contouring and the advantages and disadvantages of each RT modality in skin, are important to know. How skin reacts to RT when the skin is the target and the importance of fractionation is essential knowledge. Second, the RO needs to understand the needs of the skin stakeholders. These include those in the department who look to them for leadership. It includes those outside the department, that is, patients and other skin carers who could be future colleagues and even referrers. Third, the RO needs to use much needed research as a way to bring the disparate skin caring community together through completing high-quality research to guide therapy and post-treatment care.
\end{abstract}

Keywords: experience, skin, skin cancer, radiotherapy, practice, benign, multidisciplinary, care, Australia
Volume 7 Issue 6 - 2020

\author{
Gerald B Fogarty, ${ }^{1,2}$ David RH Christie, ${ }^{1,3}$ \\ Brad Wong, ${ }^{1,4}$ Esther Sim, ${ }^{1,5}$ Evan Ng, ${ }^{1,6}$ lan \\ Porter, ${ }^{1,7}$ Tuan $\mathrm{Ha},{ }^{1,8}$ Andrew E Potter ${ }^{1,9}$ \\ 'GenesisCare Australian Skin Tumour Reference Group \\ ${ }^{2}$ GenesisCare, Mater Sydney Hospital, Crows Nest NSW, \\ Australia \\ ${ }^{3}$ GenesisCare, Gold Coast Hospital, Tugun QLD, Australia \\ ${ }^{4}$ Genesis Care, Nambour Treatment Centre, Nambour QLD, \\ Australia \\ ${ }^{5}$ GenesisCare, Casey Radiation Oncology Centre, Berwick VIC, \\ Australia \\ ${ }^{6}$ Genesis Care, Shenton House, Joondalup WA, Australia \\ ${ }^{7}$ GenesisCare, Cabrini Hospital, Malvern VIC, Australia \\ ${ }^{8}$ GenesisCare, Rockhampton Hospital, Rockhampton QLD, \\ Australia \\ ${ }^{9}$ GenesisCare, St Andrew's Hospital,Adelaide SA, Australia
}

Correspondence: Gerald B Fogarty, GenesisCare, Department of Radiation Oncology, Mater Hospital, P.O. Box 1003, Crows, Nest, NSW, 2065, Australia,

Email gerald.fogarty@genesiscare.com Received: November 09, 2020 | Published: November 19,
2020

\section{Introduction}

Skin is the largest organ, and can suffer from a multitude of pathologies, including malignancy. The successful treatment of skin disease decreases morbidity and mortality. As we interface with the world through our skin, treatment that has the least impact on function and cosmesis should give a better quality of survivorship. Radiotherapy (RT) conserves tissue. In areas where tissue loss can have a significant impact on function and cosmesis, e.g. head and neck (Figure 1), RT could be preferred over other modalities that remove tissue yet claim to be the gold standard. ' High-quality research, especially comparative randomised controlled trials (RCTs), is needed to guide therapy and post-treatment care. Modern RT has a bright future in the treatment of skin disease. RT has improved on two fronts in the past decade. First, advances in engineering and physics have delivered better conformality and homogeneity of the radiation dose throughout the thin targets of diseased epidermis and dermis, ${ }^{2}$ with less transmission through surrounding normal tissue (Figure 2). Second, a better understanding of the different radiobiologies of tumour and normal tissue has led to personalised radiation dose prescriptions. ${ }^{3}$ This combination has enabled RT volumes to be finessed and for doses and fractionation patterns to fit the clinical scenario. The result is a patient journey that delivers more cure and less side effects in normal tissue, leading to better quality survivorship. The number of skin conditions amenable to RT is therefore growing.

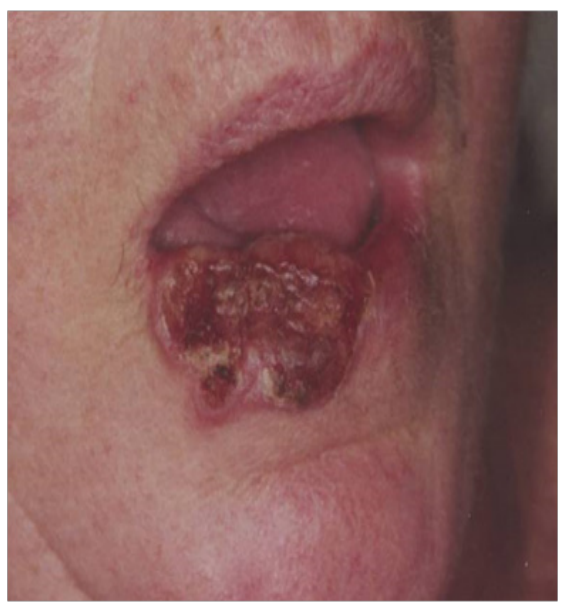

Figure I Radiotherapy (RT) has similar cure rates to surgery but also conserves tissue. Logically, it would be a better option for skin disease especially in areas where tissue loss can have a significant impact on function and cosmesis. (A) Large cutaneous squamous cell carcinoma (cSCC) of the lower lip at presentation. 


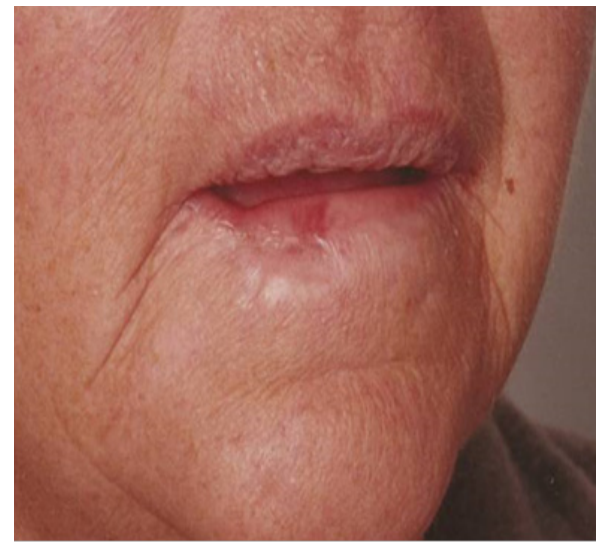

Figure IB Six months after treatment with RT alone.

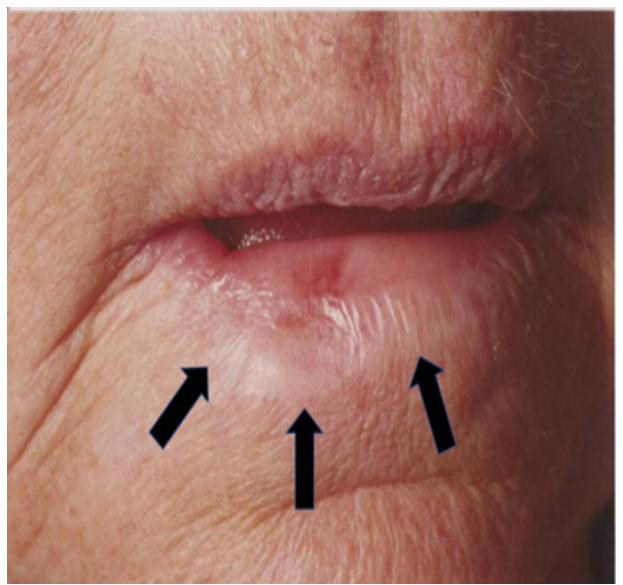

Figure IC Close up view. Arrows indicate the normal tissue that was within the tumour bulk. This tissue at least would have been sacrificed by surgery.

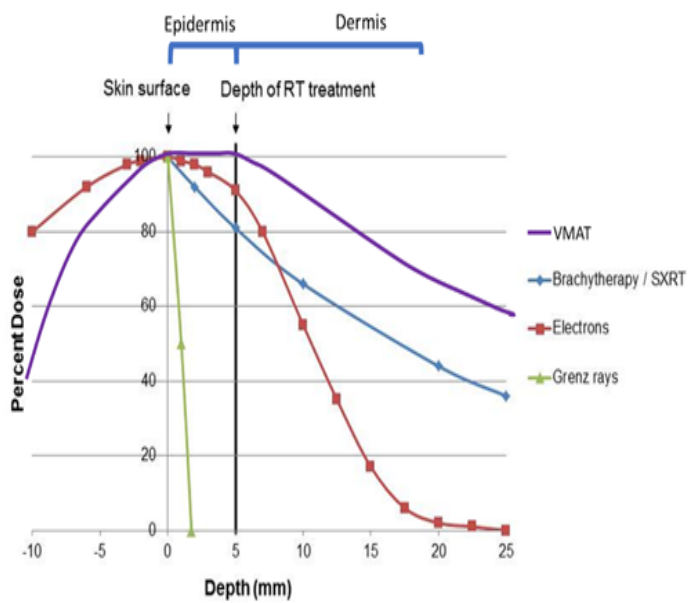

Figure 2 Advances in engineering and physics have delivered better conformality and homogeneity of the dose of radiation throughout the thin targets of diseased epidermis and dermis with less transmission through surrounding normal tissue. Schematic only.

This figure shows the depth doses of different RT modalities through the first few layers of skin. The thin black vertical line at zero represents the skin surface. The thick black vertical line represents $5 \mathrm{~mm}$ into tissue, the deepest point that skin appendages penetrate, so this area can be classed as the volume that contains the epidermis. ${ }^{4}$ Megavoltage modalities (Purple line=volumetric modulated arc therapy [VMAT] and red line = electrons [MeV]) need build up (BU) or "bolus" to ensure full dose to the surface.

Patients consider skin disease to be important in terms of quality survivorship. Payers also consider skin disease to be important. The cost of treating skin disease is increasing. According to the Australian Institute of Health and Welfare, the estimated total treatment cost for non-melanoma skin cancer (NMSC) during 2000-01 was \$AUD 264 million, in 2010 it was \$AUD 511.0 million, and in 2015 \$AUD 703 million. ${ }^{5}$ Many current treatments are not durable, ${ }^{6}$ and there are the costs, both financial, social and psychological, of repeat treatments. These costs are often based on less than optimal research quality and are evaluated on short term endpoints. ${ }^{7}$ However, in some RT departments, skin may not be viewed as a serious subspecialty. This can occur even in Australia, the country with the highest incidence of skin cancer, ${ }^{5}$ and yet the nation should take responsibility for developing level one evidence to justify RT in skin. Skin cancer is so prolific in Australia that statistics are not collected by government agencies which may, inadvertently, convey the message that perhaps skin is not as important as other tumour streams. RT has featured in the treatment of skin disease for decades. Initially, it consisted mainly of superficial radiotherapy (SXRT) prescribed and supervised by nonradiation oncologists. At this time, RT was in its infancy and radiation oncologists (ROs) were not engaged in skin. The reasons for a decrease in the utility of RT in skin included decreased reimbursement in real terms, increased regulatory requirements for radiation quality control, improvements in the efficacy of topical and systemic therapies, and the evolution of better surgical techniques. Furthermore, the radiobiology of fractionation was not known and hypofractionation delivered inferior late effects that were dominated by fibrosis, giving RT a bad name (Figure 3).

Now, significantly improved modern RT has a chance to reenter the arena. Like other indications for which RT has become standard therapy, the key drivers will be high quality research ${ }^{8}$ and patient advocacy, ${ }^{9}$ and these need to be supported by effective RO leadership. Motivated RO leaders are needed to create a specific niche for skin RT amongst a crowded field of skin treatment modalities. The purpose of this article is to share experiences from ROs who treat skin with modern RT, with those who may be interested in skin as a RT subspecialty. This article is set out in three parts. First, suggestions and practical education are provided to help the RO offer a high-quality service. Second, how to identify, understand and move forward with key stakeholders is discussed in the context of growing a successful high-quality skin practice. Third, emphasis is placed on the need for high-quality research to guide therapy and post treatment care, and how it can bring the disparate skin treatment community together. ${ }^{10}$

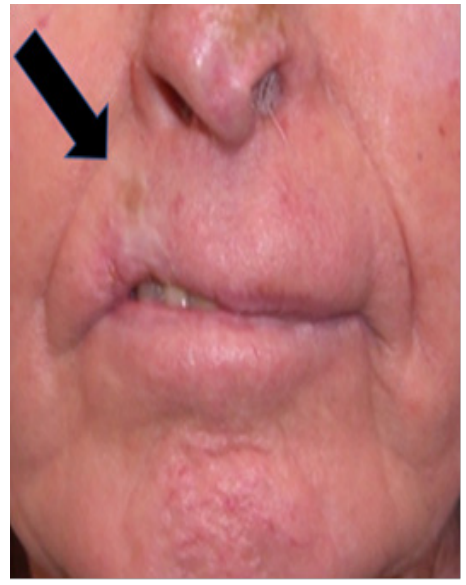

Figure 3 In the early days of skin RT, the radiobiology of fractionation was not known. Hypofractionation delivered inferior late effects dominated by fibrosis, giving RT a bad name. (A). RT was given in this case in a hypofractionated manner, probably $36 \mathrm{~Gy}$ in 6 fractions at 2 fractions per week, to a lesion on the right upper lip. RT has cured the cancer, but years later has caused in-field fibrosis, resulting in hypopigmentation, telangiectasia, thinning of the lip and cicatrisation as indicated with the black arrow. 


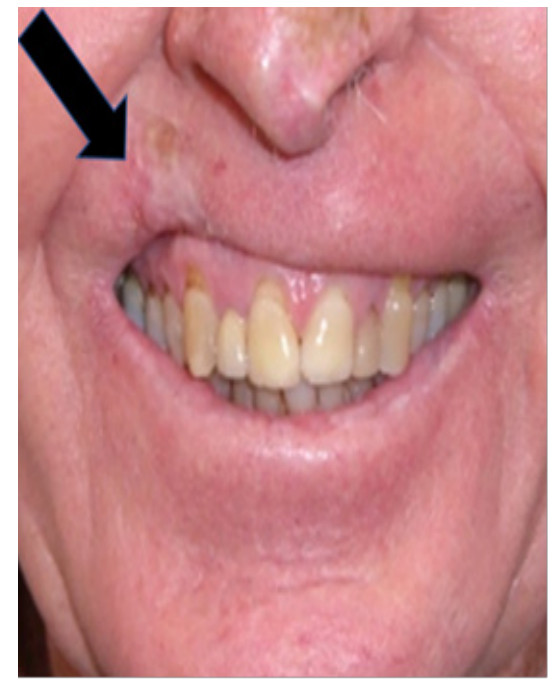

Figure 3B Cicatrisation, indicated with the black arrow, has impacted this person's smile resulting in a poor functional and cosmetic outcome.

\section{Part A: growing a high-quality service}

Building a skin RT practice is like any relationship - it is about earning trust. Trust is won by offering a consistently high-quality service. This is a long-term game and takes years to achieve but can be done with persistence, evidence-based belief in the modality and effective communication (Figure 4). Ultimately, the aim is to increase referrals by appropriately positioning RT as a treatment option and conveying the efficacy of skin RT amongst referring doctors. Table one shows the growth of RT referrals for the treatment of skin cancer of the ear over time in one Australian centre. Note that the ratio of definitive to adjuvant intent increases over time as RO-provided education enhances the referrers knowledge of the efficacy of RT for gross disease. From the patient perspective, this avoids the need for two treatment modalities and provides better survivorship due to tissue conservation (Table 1). Establishing a quality service starts with the RO themselves. What a skin RO does clinically is summarised in Table 2. A skin RO needs to know how to assess, prescribe, plan, treat and follow up on the outcomes for skin patients. Patient assessment includes taking an appropriate history, performing a physical exam and processing relevant investigations in order to decide whether RT is indicated or not.

\section{Referrals to RO for nasal BCC and CSCC over a 10- year period}

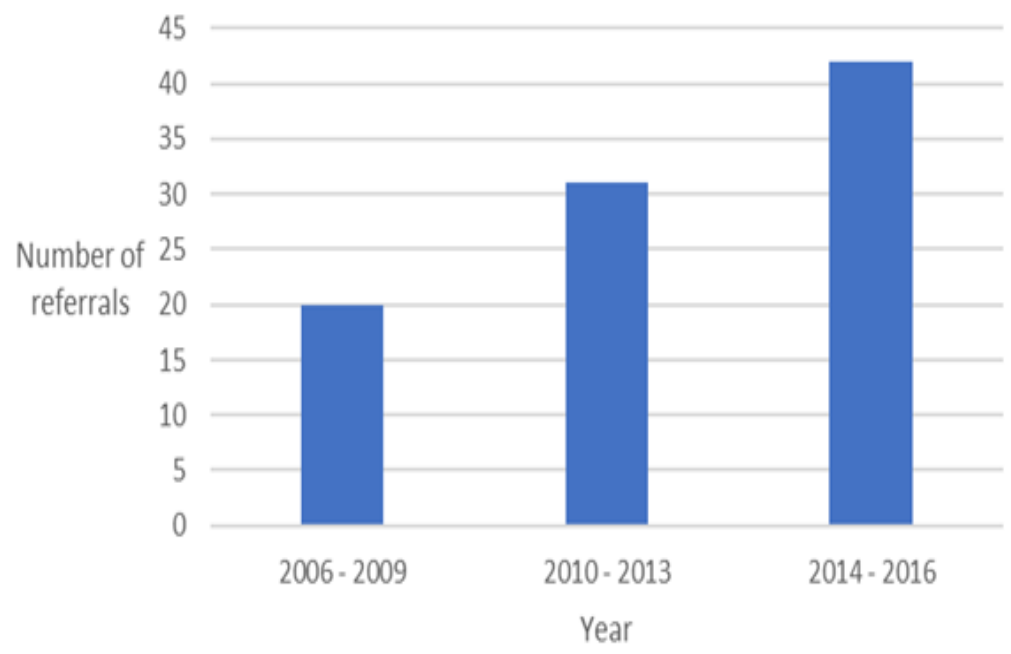

Figure 4 Growth in referrals for RT to the nose over time.Achieving growth takes a long time and reflects the time it takes to build trust within the referral base by offering a high-quality service. ${ }^{10}$

Table I Growth of RT referrals for ear lesions over time. Note that the ratio of definitive to adjuvant intent increases over time as the referrer's knowledge and confidence in the efficacy of RT grows (Anthony Tanous unpublished data)

\begin{tabular}{llll}
\hline Year & $\begin{array}{l}\text { Definitive Intent } \\
\text { (number of patients) }\end{array}$ & $\begin{array}{l}\text { Adjuvant Intent } \\
\text { (number of patients) }\end{array}$ & Ratio of Definitive/Adjuvant Intent \\
\hline $2007-2011$ & 6 & 9 & 0.66 \\
$2012-2016$ & 24 & 18 & 1.33 \\
$2017-$ present & 28 & 8 & 3.5 \\
Total & 58 & 35 & 1.7 \\
\hline
\end{tabular}


Table 2 What skin ROs do

\begin{tabular}{|c|c|c|c|}
\hline What Skin ROs do & Interact with & Decisions RO needs to make & Where this happens \\
\hline See new patients & Patient and family & Decides if RT is indicated & Clinic \\
\hline Planning & RT Staff, Nursing & $\begin{array}{l}\text { Decides if the plan is acceptable } \\
\text { Decides on concurrent treatments }\end{array}$ & RO department \\
\hline On treatment reviews & Patient and family, RT staff, Nursing & $\begin{array}{l}\text { Assesses the development of acute effects } \\
\text { Decides whether to continue, change or cease RT }\end{array}$ & RO department \\
\hline Follow Up & $\begin{array}{l}\text { Patient and family, Multidisciplinary } \\
\text { colleagues }\end{array}$ & $\begin{array}{l}\text { Decides on whether treatment endpoints have been met, e.g. } \\
\text { whether disease is controlled, or symptoms palliated, and if } \\
\text { there are any late effects that can be treated }\end{array}$ & Clinic \\
\hline
\end{tabular}

\section{Patient assessment}

\section{History}

Obtaining a complete history of the patient, the tumour and its treatment are fundamental, especially details that may impact the dose of RT and the volume to be treated.

\section{Patient factors}

Specific patient factors in skin RT include a history and duration of any immunosuppression e.g. previous transplant and current levels of immunosuppressive medicines, chronic lymphocytic leukaemia or treatment for human immunodeficiency virus infection (HIV). Skin cancers grow more quickly and metastasise more often in the immunosuppressed. ${ }^{11}$ Often these patients are more radiosensitive for reasons yet to be elucidated. ${ }^{12}$ Previous skin cancers, their treatment and outcomes, may lead to fine-tuning of the radiation dose. Prior radiation to the index site will affect the radiation prescription, often leading to more hyperfractionation. The medications history needs to include asking about radiation sensitising drugs such as methotrexate and hydroxyurea. If these are to be continued during RT, a close eye must be maintained on the development of acute toxicity as the dose may need to be reduced given the radiation sensitising effect of these drugs. Standard fractionation of two Gray (Gy) or lower per day is also advised as, at this rate, side effects tend to keep pace with the dose rate.

Past and family history of skin cancer, other cancers, and unusual toxicities from previous radiation treatments in the family may lead to a clinical suspicion of a rare skin cancer syndrome (e.g. Gorlin syndrome) or radiation sensitivity syndrome (e.g. mosaics of xeroderma-pigmentosum) and may prompt a genetic review. Prior to RT, assessment of the patient's support network, especially with regards to transport to and from the treatment centre and help with dressings and activities of daily living, should be carried out. Food preparation is important as good nutrition is needed to ensure repair of normal tissues during and after RT, otherwise acute reactions can be more severe, and treatment completion may be compromised. A lack of adequate patient support may even push the treatment team to consider another therapeutic option, for example, more surgery rather than post-operative RT (PORT) to guarantee local control (LC).

\section{Tumour factors}

A specific history of tumour factors needs to record the time interval from the moment the abnormality was first detected to the present time, and note any symptoms including bleeding, ulceration, the need for dressings, or the crust-resolve-crust cycle that is typical of basal cell carcinoma (BCC). Any perineural invasion (PNI) symptoms such as pain, numbness and formication on or around the lesion need to be ascertained, as this can change the RT volume. Lymph nodes, e.g. neck, axilla, epitrochlear, groin and popliteal, should be assessed for swellings. The patient may not be aware of them or associate them with the primary disease, and previous medical attendants may not have examined them.

\section{Treatment factors}

A history of previous treatment needs to be elicited, especially previous surgery for cutaneous squamous cell carcinoma (cSCC), as recurrence is an absolute indication on the EViQ website for PORT. ${ }^{13}$ Further RT may not be possible if there is a history of previous RT and alternative therapies would need to be considered. It is important that the planning staff retrieve the previous RT plans as recurrence post RT can be field-edge recurrences and further RT may still be possible.

\section{Examination}

Examination involves inspection and palpation. Skin diseases amenable to RT cover all adult age groups. Adequate assessment of performance status is crucial to decide if a patient is fit for radical treatment. Whether the patient can actually have RT is also important to note. Those with the inability to keep still or tolerate immobilisation devices may be excluded from RT. Those challenged with claustrophobia or the inability to understand (e.g. dementia patients) may need sedation or a different RT modality, such as SXRT, which does not mandate a mask during treatment to the head and neck. A hint that the patient is more radiation sensitive is the observation of Fitzpatrick type 1 skin on examination where the patient has white skin, red hair, blue eyes, and a tendency to freckle and burn rather than $\tan .{ }^{14}$ The examination tools the skin RO needs are shown in Figure 5. "Scanning" for disease in other tumour types involves a radiology or nuclear medicine third party provider. In skin, the RO does their own scanning with a strong light, marker pen, and face mirror. The face mirror is needed so that the patient can help to delineate any facial lesions (Figure 6). The mirror also helps engage the patient into agreeing on what area should be treated which is essential for informed consent. A template can then be made of this area with the plastic protector sheet ${ }^{15}$ before the planning skin marks are erased, so that the treatment field information can be captured for planning. This avoids having to re-mark the area and having to repeat the conversation at simulation. With an experienced planning team, it may even mean that the clinician is not needed to attend simulation, improving logistics and scheduling. The ruler is used to adequately stage the primary. ${ }^{16}$ The template helps to record the primary environment, as does the camera. Persistent, or even already recurrent disease, may be seen at the edge of a graft where there was a positive margin. Experience, repeat biopsy, and often clinical involvement of the surgeon, may be needed to ensure that this is only granulation tissue. The RO mantra of covering all the surgical bed may need to be compromised depending on the patient's ability to attend for the total number of fractions required. 


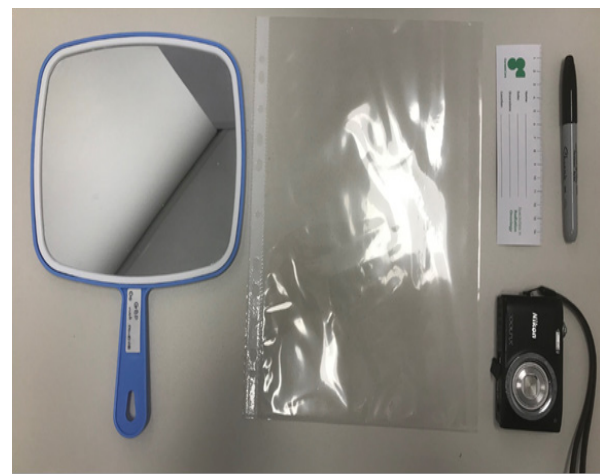

Figure 5 Tools the skin RO needs.

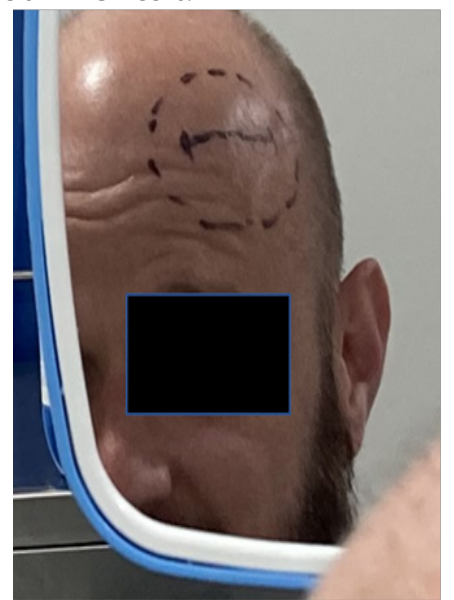

Figure 6 Use of the face mirror and markings which also help to obtain informed consent.

In this scenario, the RO has traced the area needing RT onto the patient's skin. Using the face mirror, the patient is better able to understand what is to be treated and provide informed consent. A template can then be made of the proposed treatment area before the marks on the skin are erased so that it can be captured for planning. This avoids the need to re-mark the area and to repeat the conversation at simulation.

Palpation, especially bimanual palpation of the ears, lips, nasal alar, cheeks, genitals and digits, helps to assess depth and therefore the quality of the RT beam to be used. Macroscopic recurrence may be felt under a flap or graft where there was a positive deep margin depending on the time since surgery. The RO needs to know where the positive margin is under a flap if a boost is being considered. The lesion is always at the flap vertex which is not necessarily in the middle of the operative field (Figure 7). Draining lymph node stations need to be palpated, especially the epitrochlear node in the upper limb and the popliteal node station in the lower limb, as these are often missed by physicians who are not mainstream skin carers. Pre-existing graft and flap failure should be assessed and documented, preferably with photos. ${ }^{17}$ Failure is problematic. Studies show that PORT needs to start within a certain timeframe otherwise local control and even survival can be compromised..$^{18}$ Graft and flap failure can often mandate reoperation, or healing by secondary intention, which can further delay or even invalidate PORT.

The histopathology report needs to be interrogated to adequately stage the cancer. Details of locally advanced disease include extratumoral PNI, lymphovascular invasion (LVSI), positive margins and/or invasion of other structures. Unfortunately, the data on when PORT should be given is not of robust quality, ${ }^{13}$ and often it's a case of guesswork to determine the risk of recurrence without PORT based on the relative number of favourable and unfavourable prognostic factors discovered during patient assessment (Table 3). Randomized controlled trials (RCTs) are needed in this space. Given that the RO is essentially the histopathologist's customer, the RO can leverage this position to obtain better histopathology reporting, even synoptic reporting, in the best interest of all. ${ }^{19}$ If the RO decides to offer RT, then radiation decisions need to be made (Table 4). The most important decision is to decide what the intent of therapy is. Radical or curative intent often mandates weeks of fractionated treatment, significant acute effects and a long follow up program. Palliative intent implies treating or avoiding a symptom, with a short fractionation pattern and minimal acute effects (Figure 8).

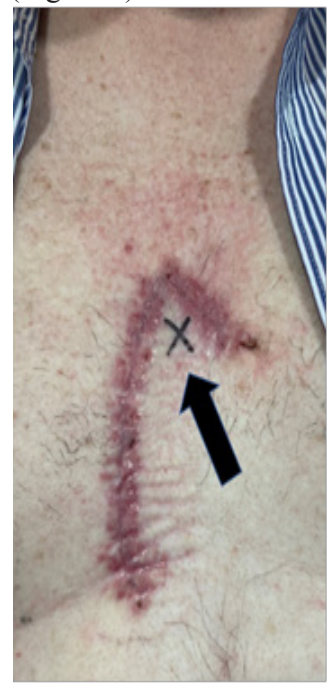

Figure 7 Position of the lesion bed under a flap. The black arrow points to a cross in this flap repair of a lesion on the anterior chest. The patient identified this as the position of the original lesion. The lesion bed is always at the flap vertex which is not necessarily in the middle of the operative field.

Table 3 Patient, tumour and treatment factors influencing the decision to prescribe local RT for skin disease

\begin{tabular}{|c|c|c|}
\hline Factor & & $\begin{array}{l}\text { Presence of this } \\
\text { factor may favour: }\end{array}$ \\
\hline \multirow[t]{4}{*}{ Patient } & Immunosuppression & RT \\
\hline & $\begin{array}{l}\text { Challenged mobility/home } \\
\text { support/ability to tolerate } \\
\text { immobilisation/ability to attend } \\
\text { fractionated therapy }\end{array}$ & $\mathrm{Rx}$ other than $\mathrm{RT}$ \\
\hline & Low need for function/cosmesis & $\mathrm{Rx}$ other than $\mathrm{RT}$ \\
\hline & $\begin{array}{l}\text { Radiation sensitivity syndrome/ } \\
\text { drugs }\end{array}$ & $\mathrm{Rx}$ other than $\mathrm{RT}$ \\
\hline \multirow[t]{5}{*}{ Tumour } & Recurrent disease & RT \\
\hline & $\begin{array}{l}\text { PNI, LVSI especially if extra } \\
\text { tumoral }\end{array}$ & RT \\
\hline & Close/positive margin & RT \\
\hline & High risk site - e.g. ear & $\mathrm{RT}^{16}$ \\
\hline & Depth over $4 \mathrm{~mm}$ & $\mathrm{RT}^{38}$ \\
\hline \multirow[t]{2}{*}{ Treatment } & Previous RT to index site & Rx other than RT \\
\hline & Failed graft/flap & Rx other than RT \\
\hline
\end{tabular}

LVSI, lymphovascular space invasion; mm, millimetre; PNI, perineural invasion; RT, radiotherapy; Rx, treatment 
Table 4 Radiation decisions that skin ROs need to make

\section{Decisions skin ROs make}

I Intent of therapy - radical or palliative

2 To prescribe RT or not - consider alternate therapies e.g. surgery

3 Volumes of cancer to be treated and normal tissue to be avoided

4 What RT modality(ies) gives best conformality/homogeneity of dose to the intended volumes?

5 What total dose is needed? What fractionation?

6 What are the concurrent concerns/treatments, if any? e.g. chemotherapy, regular dietician review, ophthalmologist review, dentist review prior to RT

7 What intensity of on-treatment review and follow-up?

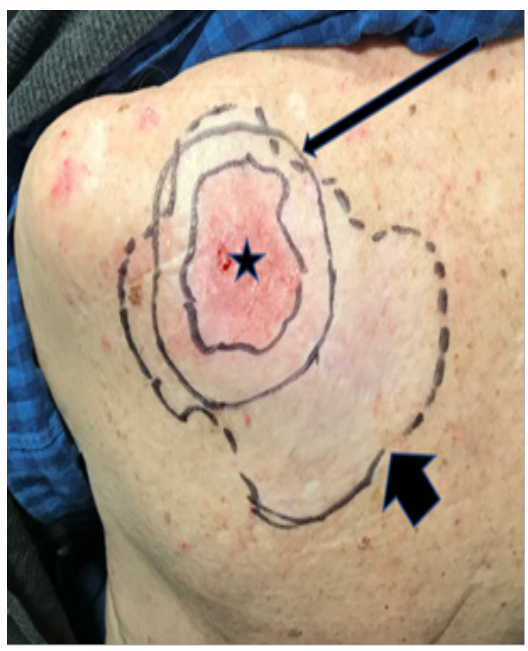

Figure $\mathbf{8}$ The most important decision is the intent of therapy.

This wheelchair bound man with a poor performance status had a BCC on his left shoulder and back that had gross subcutaneous morpheaform invasion over a large area (short black arrow). The reason he came for treatment was to stop the ulceration (black star) of part of the BCC that caused bleeding, pain, odour and required daily dressings. This was adequately treated by an electron field which is indicated by the long black arrow. This field was treated with $18 \mathrm{~Gy}$ in 3 fractions at one per week with no symptoms and did not require dressings three months later.Treatment of the whole lesion to tumour resolution would have taken weeks of daily RT.

The RO needs to explain the rationale, process, expected efficacy and side effects to the patient in order to obtain informed consent. Planning marks can be made on the patient's skin with washable ink. The treatment area can be agreed upon by both the RO and patient. This is important as, for example, the waxing and waning of BCC may be in a waning phase and the patient may need to identify the actual size from memory, or from past photos, which will affect the RT field size. For head and neck patients a face mirror can be invaluable. A letter is then written to the referrer. The quality of correspondence between the $\mathrm{RO}$ and the referrer is crucial as it can indicate the quality of the service. In general, the quality of correspondence in the care of skin patients can be improved and will benefit from the holistic oncological approach that the RO is trained in. Table 5 outlines the type of information that correspondence could contain, and Table 6 suggests the content of an end-of-treatment letter. The RO supervises the planning and treatment, interacting with the nursing and allied health staff, to ensure prescription completion with as few side effects as possible.
Table 5 Suggested content of correspondence to the referrer following initial consultation.

\begin{tabular}{|c|c|}
\hline Paragraph & Content \\
\hline & Salutation and acknowledgement of thanks for the referral \\
\hline I & $\begin{array}{l}\text { Patient factors: gender, age, domicile, occupation, mobility, } \\
\text { support }\end{array}$ \\
\hline 2 & $\begin{array}{l}\text { Reason for referral: RT for definitive, adjuvant, salvage or } \\
\text { palliation }\end{array}$ \\
\hline 3 & $\begin{array}{l}\text { Tumour factors: anatomical location, history, histology, } \\
\text { stage }\end{array}$ \\
\hline 4 & $\begin{array}{l}\text { Past medical history of relevance: immune suppression, } \\
\text { concurrent medication }\end{array}$ \\
\hline 5 & $\begin{array}{l}\text { Examination: patient's ability to have RT; tumour, regional } \\
\text { nodes and PNI signs }\end{array}$ \\
\hline 6 & $\begin{array}{l}\text { Whether RT is possible and, if so, what modality, dose and } \\
\text { fractionation are planned }\end{array}$ \\
\hline 7 & $\begin{array}{l}\text { Document discussion of rational, process and side effects } \\
\text { of therapy and whether informed consent was obtained }\end{array}$ \\
\hline 8 & Brief overview of schedule for planning and treatment \\
\hline 9 & $\begin{array}{l}\text { Expected acute effects: when and how they will be } \\
\text { managed }\end{array}$ \\
\hline \multirow[t]{3}{*}{10} & Expected oncological outcome, preferably with a reference \\
\hline & $\begin{array}{l}\text { Thanks for the referral; appropriate farewell greeting and } \\
\text { signature }\end{array}$ \\
\hline & $\begin{array}{l}\text { Appropriate carbon copy recipients, especially to the GP, } \\
\text { and the institution, if relevant }\end{array}$ \\
\hline
\end{tabular}

\section{Planning}

RT planning proceeds in the department and is the first time the patient meets the rest of the skin radiation team. This is an opportunity for the skin RO to bring the rest of the team on the journey of providing a high-quality skin RT service. If the initial consultation is in the same location as the planning team, having a tentative planning session ready is convenient for all, especially for patients with challenged mobility or those who rely on others for transport, as this saves another trip. During the initial consultation, if the RO has traced the area of skin needing RT on the patient and informed treatment consent has been obtained for this area, having the planning team create an immediate template before the marks are erased is invaluable to the efficiency of the planning process. ${ }^{16}$ The precise area can be captured for planning, avoiding the need to re-mark the area and to repeat the conversation at simulation. Selection of the treatment modality needs to be made early in the planning journey. Selection is determined 
by the type of modalities available on site in the treatment centre. Networked centres may have a spread of modalities between locations and this may involve patients having to go to another site to access the modality best suited to their condition. This is an important point to address in the initial consultation. Knowing the percentage depth dose of the different modalities and energies is fundamental to high quality skin RT (Figure 2). The skin RO needs to keep up with new treatment modalities and techniques together with their toxicities..$^{20,21}$

Simulation then occurs. It is the ROs responsibility to ensure that simulation goes well. It pays to be present to avoid re-simulation. Treatment positioning and immobilisation are performed to enable exact contouring. The RT team take a template of the field ${ }^{15}$ which should be stored for use during treatment and follow-up. Photos are taken of the RO's marks and stored in the electronic patient record (EMR) prior to wiring the marks. Wiring refers to the imposition of wire onto the marks made by the RO. Wiring is necessary so that the marks can be captured faithfully by the planning $\mathrm{CT}$ to aid with contouring. The RO needs to understand the subtleties around bolus placement and the possibilities and importance of air gaps between bolus and skin (James Hellyer personal communication). On site physics enables a physics opinion to be available at planning. Physicists can help inform the treatment modality decision, e.g. electrons or volumetric modulated arc therapy (VMAT) for field therapy; between electrons, three-dimensional conformal RT (3DCRT), or superficial radiotherapy (SXRT) for discrete nose lesions. Having a member of the physics team present at planning can also address the need for in vivo dosimetry (IVD), a quality assurance practice that particularly lends itself to skin. The RO, physics and RT staff can agree on exactly where IVD needs to occur supported with proper documentation including photos and template. ${ }^{15}$ SXRT, along with the use of bolus and shields (e.g. internal eye, teeth, posterior ear and nasal shields), can be inspected for positioning during the first week. Table 6 describes a check list at simulation for ROs. ${ }^{22}$

Table 6 RO check list at simulation

\section{RO to consider}

Patient position: adequate for the proposed treatment modality? Contralateral limb out of beam?

2 Patient immobilisation: comfortable, stable, reproducible?

3 All marks carried out including CTV, SIB areas and crosses for IVD; fields for SXRT and electrons

4 Photos and templates completed, and all stored for future reference

5 RO marks wired appropriately to assist with contouring

6 Bolus placement: adequate, comfortable, stable, reproducible? (if done during simulation)

\section{RO to consider}

7

CT protocol followed adequately including superior and inferior border and scan interval

8 Acquired CT inspected for adequacy for contouring purposes

Wires and marks removed from the patient prior to discharge from planning

CTV, clinical target volume; IVD, in-vivo dosimetry; SIB, simultaneous integrated boost

SXRT access is invaluable to a skin RO for many reasons. SXRT machines have low quality beams so there is less need for shielding. The head of the machine is easily moveable so that a patient can be treated in a wheelchair or bed, meaning that difficult transfers are avoided. The treatment applicator has a transparent perspex ending so the treatment field can be clearly seen when the cone is in position, unlike an electron applicator that needs BU. The SXRT applicator can be rested on the treatment area ensuring that the correct field is treated. Masks are not necessary, saving cost and treatment set up time. SXRT treatment can be viewed through lead windows from the control console where eye contact with the patient can be maintained so that even patients with attention deficits, especially dementia, can be treated. Some SXRT machines are transportable so that treatment can be brought to the bedside.

As more skin patients are treated in the department, the RT team will become more autonomous, but new staff with little experience of skin RT may forget to wire, create a template, or sufficiently photograph the setup, which may sometimes lead to re-simulation. A networked centre can often mean that the planning is done off-site by a planner that is not versed in the subtleties of skin planning. Clear protocols can help. It is important to take care when contouring the true patient outline and the wires and volumes to be avoided (e.g. previous RT fields) as the contours for treatment depend on these. Simulation marks need to be cleaned off the patient after planning is complete. Contouring can be challenging and time consuming especially for large thin areas. ICRU ${ }^{22}$ guidelines and specific protocols ${ }^{23}$ should be followed. Generic automatic contouring and scripting should be checked for each case. For patients having multiple sites treated simultaneously, scripts for each area need to be generated and checked. The treatment prescription for different areas on the same patient can differ. Contouring can include avoidance volumes to guide planning. Megavoltage and even higher energy SXRT can penetrate deeply, so the dose to organs such as parotids, lacrimal glands, and hippocampi ${ }^{24}$ needs to be considered to avoid unwanted late effects.

The prescribing of skin RT is personalised. Clinical skin scenarios differ so much that skin prescriptions can be hard to automate. A 25-year old with a keloid of the lower pinna will have a different prescription to that of an 80-year old with a positive margin SCC in the same anatomical place. Standard fractionation of 2 Gy per day or less means that the acute radiation side effects occurring at any given time in treatment correspond to the dose delivered at that stage. ${ }^{25}$ Hypofractionated courses are common in skin cancer and often have a component of acceleration so that toxicity can peak days after all the dose has been delivered. This means titration to the acute reaction of the day cannot be done. Standard fractionation and regular physician review enable dose titration, and perhaps a lower overall dose may be all that is needed. ${ }^{26}$ Skin does lend itself to treating to a reaction rather than just to a set dose, which may not be enough, even in insitu disease. ${ }^{27}$ In situ disease seems to handle a break without loss of oncological control. ${ }^{12,28}$ Plan acceptance then takes place. This demands attention to detail, notably to PTV coverage and doses to organs at risk (OARs). Plans may require peer review, especially for those seeking accreditation for new techniques. This is a great opportunity to learn and increase confidence from mentors who are keen to see more ROs accredited. This also grows the unity, collegiality and redundancy within the networked RO team. Documentation of peer review is advised and is also helpful for continuing professional development (CPD) and the rare medicolegal case. On treatment reviews (OTRs) are important for skin cancer patients. A review in the first week is an opportunity to go over the plan document and to show the patient how the tumour is being covered and how OARs are being avoided. This builds confidence and trust, not only with the patient but also with the patient's carer who is often a relative. If this cannot be done face-to-face, a phone call reassures patients that they are being closely 
followed. The OTR is the logical moment to describe the side effects that may occur during the following week of treatment. This aligns patient expectations with what will happen, winning confidence and a desire to complete the script despite worsening side effects. Regular OTRs are an opportunity to titrate the dose as some skin patients are inexplicably RT sensitive. ${ }^{14}$

OTRs are also an opportunity to assess if further IVDs are needed to check the accuracy of dose delivery if normal in-field skin is not reacting as expected. Furthermore, OTRs provide an opportunity to facilitate communication amongst the entire treatment team so that the RO knows about any unusual events that have been noted by other staff. An example of this is a mask becoming loose, which can be a sign of weight loss and which may result in acute effects not healing within the expected timeframe. Another example is tightening of the three-dimensional printed bolus on a lower leg, heralding the initiation of lymphedema and suggesting a treatment break to avoid pain and geographical miss.

\section{Skin changes and care during RT treatment}

Skin care during the treatment journey is important and patients will ask about it. ROs and nurses need to cooperate well here. Initially the only skin care needed is moisturisers. This is because the first organs within the in-field normal skin to suffer will be the skin appendages sweat glands and hair follicles that provide natural moisture and have significant populations of radiation sensitive normal stem cells. In field normal skin has four grades of reaction. These are described by various guidelines. ${ }^{29,30}$ They can be explained based on normal acute inflammation caused by RT and the radiobiology of normal skin. They are summarised in Table 7. Grade 1 is erythema and begins when about 10 Gy has been delivered when 2 Gy daily fractions are used five days a week. It results from inflammation causing vasodilation in the dermis. Moisturiser is indicated. Grade 2 is dry desquamation and begins when about 30 Gy has been delivered when 2 Gy daily fractions are used. It is caused by a lack of moisture being produced by the skin due to the death of moisture-producing stem cells in the hair and sweat glands. This moisture binds together the normal covering of dead keratinocytes on the skin surface. No moisture means that it begins to flake off. Moisturiser is indicated. Grade 3 is wet desquamation and begins when about $50 \mathrm{~Gy}$ has been delivered when 2 Gy daily fractions are used. Wet desquamation is due to a lack of normal skin stem cells. As there are not enough stem cells present to grow cells to seal the dermis, interstitial fluid begins to ooze through the epidermis. A gel dressing is now needed to encourage the surviving cells to cover the gaps. The open skin is at risk of infection and needs expert nursing care and regular dressings even when the RT is finished. Plastic cling wrap is an economical and ideal way to keep wet dressings in place and to stop them from spoiling clothes. It can even be used in the department when a patient needs to move from one treatment area (e.g. machine) to another (e.g. nursing or doctor review) through public areas in the department (Figure 10).

Table 7 Changes in normal skin during RT using 2 Gy fractions five days a week

\begin{tabular}{lllcr}
\hline Grade & Observation & Cause & At Gy & Care needed \\
\hline I & erythema & vasodilation in the dermis & I0 & Moisturiser \\
2 & dry desquamation & $\begin{array}{l}\text { lack of moisture being produced by the skin due to death of } \\
\text { moisture producing stem cells in hair and sweat glands }\end{array}$ & 30 & Moisturiser \\
3 & wet desquamation & $\begin{array}{l}\text { lack of normal skin cells being able to seal the dermis, and } \\
\text { interstitial fluid begins to ooze through the epidermis. }\end{array}$ & 50 & gel dressing \\
\hline
\end{tabular}

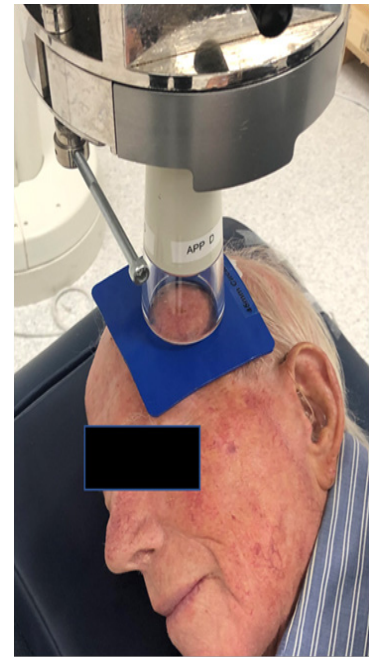

Figure 9 SXRT access is invaluable to a skin RO for many reasons.

SXRT machine in use showing that the head of the machine is easily moveable so that a patient can be treated easily.The treatment applicator has transparent perspex ending so the treatment field can be clearly seen when the cone is in position. The SXRT applicator can be rested on the treatment area to ensure that the correct field is treated. This removes the need to use a mask.

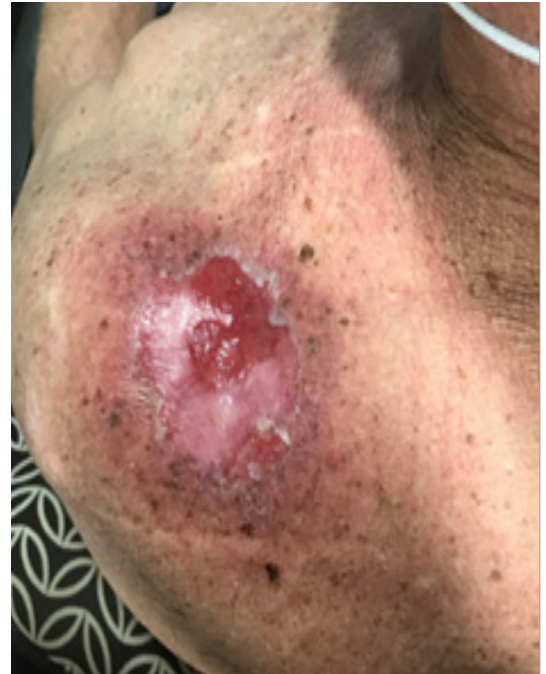

Figure 10 Use of cling wrap as a dressing for open wounds. (A) View from the superior aspect of a BCC on the tip of the shoulder being treated definitively with RT. This patient in the last stages of RT and ulceration with tumorlysis is present. In the surrounding normal skin, there is skin erythema and desquamation. 


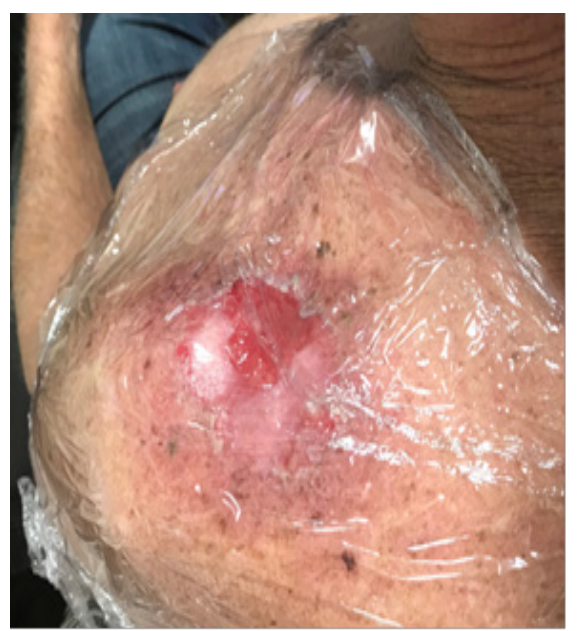

Figure I0B Gel dressing is kept moist and clothing over the top is kept dry with the use of cling wrap.

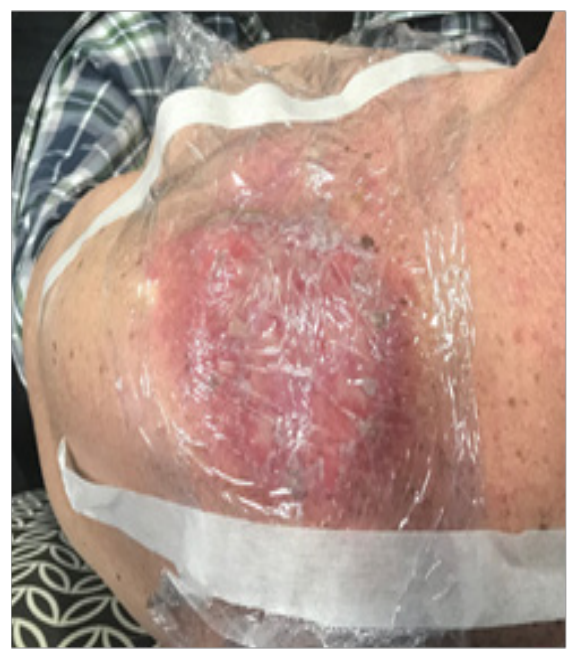

Figure I0C The cling wrap needs to be taped to skin outside the treatment field. Otherwise, when taking off the tape, healing skin can be disrupted, and acute effects may be worse than if the tape had been applied infield.

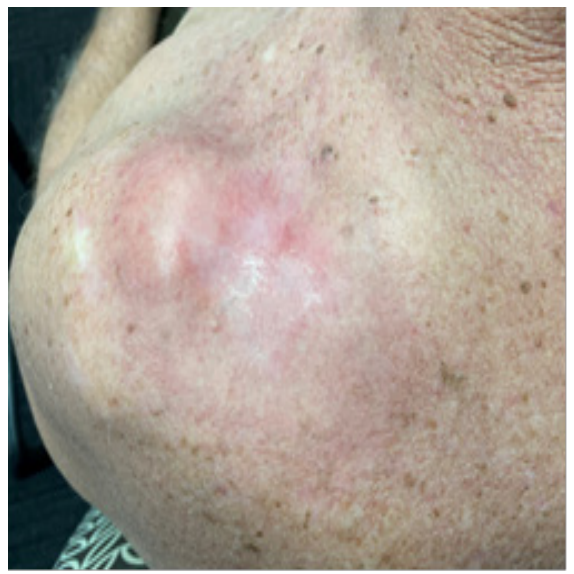

Figure IOD Shoulder wound healed three weeks after cessation of RT.

Complete re-epithelization occurs 10-14 days post RT in the normal healthy patient who is well nourished (Figure 11). Grade 4 is skin necrosis and is caused by irreparable death of skin stem cells. This is often associated with ulceration and pain. It is important to avoid this. It occurs in normal skin at $70 \mathrm{~Gy}$. Surgery may be needed to palliate the pain. The corresponding changes in cancerous cells are different. Tumour responds quickly to RT with tumorlysis producing a yellow necrotic substance that is dead tumour and not pus. Antibiotics will not help. Normal in field skin seems unaffected (Figure 12). In the first few weeks of treatment peri-tumoral bleeding can occur. This occurs because the tumour is being destroyed at a faster rate than normal skin can grow to cover the gap. Bleeding results because of the continuing irradiation. This is not wet desquamation and is peculiar to scenarios when skin is the target. This is temporary and will recover once the RT stops, allowing normal skin to rapidly close the gap (Figure 13). There can be a period mid-way through treatment when the macroscopic cancer is gone and there are minimal acute changes in normal in-field skin. It is tempting to contemplate ceasing treatment. ${ }^{3}$ Some have unexplained radio sensitivity. Further research is needed to see if RT can be stopped at this point (Figure 14). At the end of treatment another letter is dictated (Table 8). For a new referrer, a phone call commenting on the successful completion of treatment can perhaps help to build bridges. RO follow up is organised when convenient for the patient and carer and usually falls a month after treatment completion. The follow up consultation is to assess the resolution of acute effects and, with definitive RT, to assess tumour response. In the meantime, nurses can help with dressings and monitor the resolution of acute toxicity, escalating any concerns to the RO when needed. Follow up can then be either delegated back to the referrer or carried on in a shared manner. Taking over the patient may be perceived poorly by the referral base and will not encourage referrals. It is also not a move in line with better patient care, as other skin carers are better trained at skin surveillance than ROs.

\section{Part B Understanding the landscape of skin stakeholders}

This section deals with other radiation craft groups essential for delivering high quality skin RT, external skin care practitioners including referrers, patients and management.

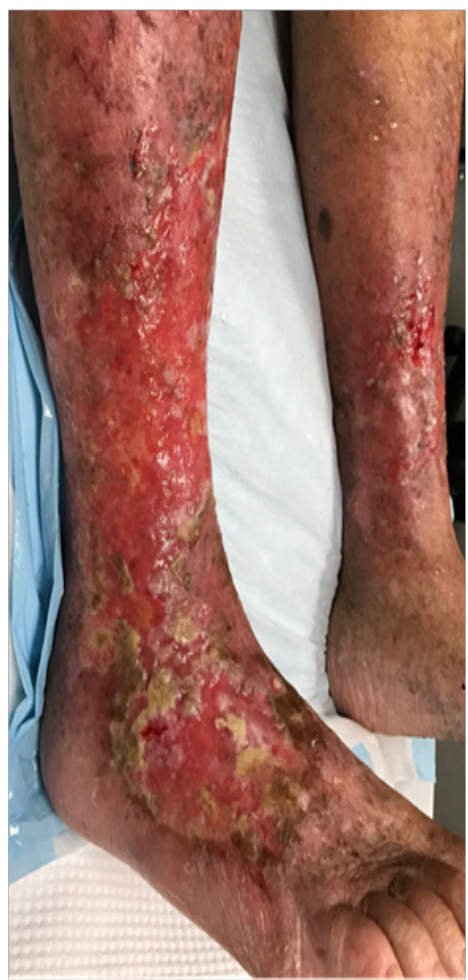

Figure I I Rapid epithelisation after RT. (A) 3 days after RT ceased. 


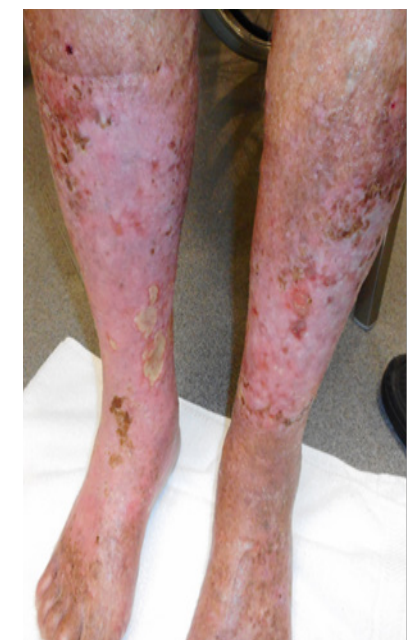

Figure IIB 12 days after RT ceased - infield re-epithelization is almost complete.

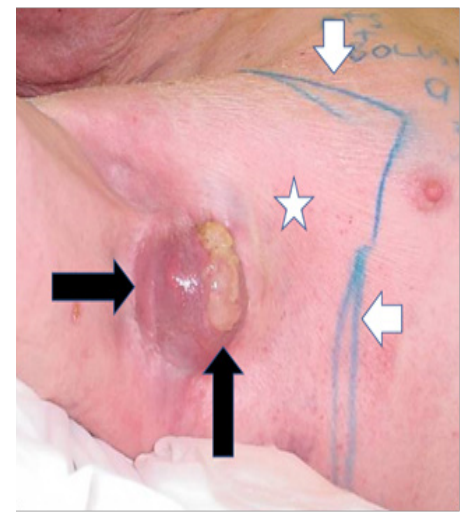

Figure 12 Tumour and normal skin react differently to RT. Definitive megavoltage radiation of an axilla and axillary mass of cSCC (black horizontal arrow) has been given so far to $27 \mathrm{~Gy}$ in $3 \mathrm{~Gy}$ fractions. The radiation field on the skin is marked by short white arrows. Normal skin receives the full dose as it is covered with bolus during treatment. In field normal skin (white star) appears unchanged. The tumour (horizontal black arrow) is suffering tumorlysis (vertical black arrow) which is shown by the yellow material on top of the cancer.

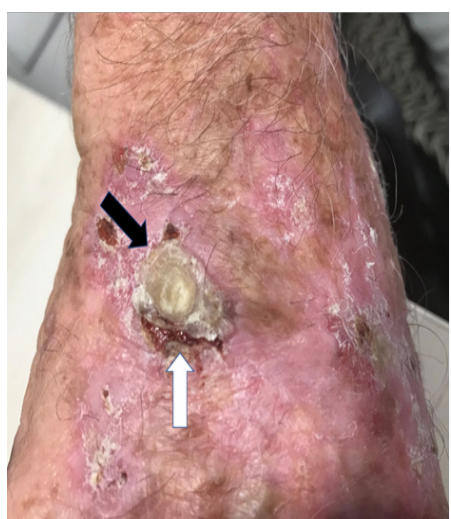

Figure I 3 Black arrow shows a skin cancer within field change on the back of a hand being irradiated. In the first few weeks of treatment peri-tumoral bleeding can occur. In this figure (white arrow) there is bleeding between the receding tumour and the normal skin. The normal skin is prevented from closing the gap because of the continuing RT.This occurs because the tumour is being destroyed at a faster rate than the normal skin can grow to cover the gap. Blood is then seen in the gap and the gap can bleed needing dressings. This is not wet desquamation. This is temporary and will recover when the RT stops, allowing the normal skin to rapidly close the gap.

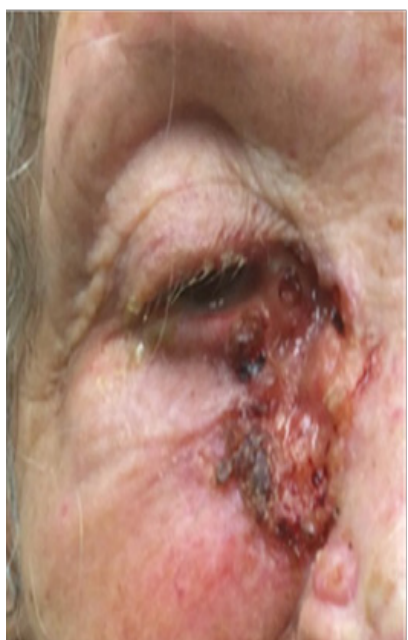

Figure I 4 Unexplained radio sensitivity. (A) Elderly lady with BCC right inner canthus.

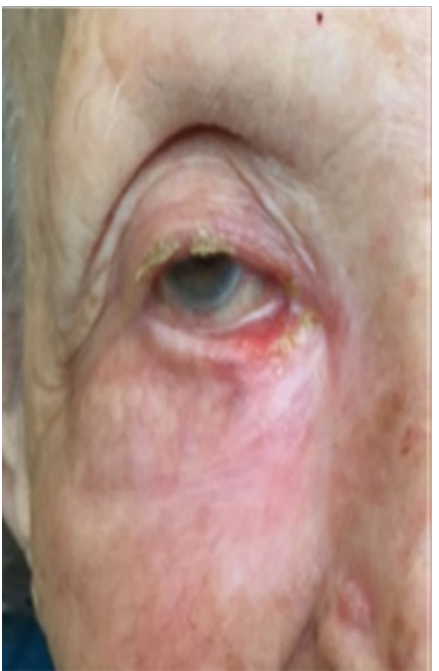

Figure I4B Eight weeks after phase one of 25 Gy in 5 fractions over a week, the first phase of the $\mathrm{ASCRT}^{3}$ showing complete response and not requiring the second phase.

Table 8 Suggested content of the End ofTreatment letter to the initial referrer

\begin{tabular}{ll}
\hline Paragraph & Content \\
\hline I & Indication for RT \\
2 & $\begin{array}{l}\text { RT details - total dose, no of fractions, phases, dates of } \\
\text { RT start and stop }\end{array}$ \\
& $\begin{array}{l}\text { Progress during RT - expected acute effects, unexpected } \\
\text { effects how managed and resolved, expected and } \\
\text { unexpected breaks }\end{array}$ \\
& Follow up plan \\
& Thanks for referral and appropriate farewell and signature \\
& $\begin{array}{l}\text { Appropriate carbon copy recipients, especially to GP, and } \\
\text { institution if relevant }\end{array}$
\end{tabular}

\section{Radiation craft groups}

The onus on having a high-quality skin RT service falls on the RO. The first craft group that a RO needs to connect with is his own RO peers in the department or network. Quality communication ensures that high-quality patient care continues when the responsible consultant is 
away. Peer support and mentoring is essential to a safe high-quality practice. Good colleagues will provide patient cover when a RO is away. If the network is big enough, cases can be discussed with an expert skin RO peer panel. This can even count for RO CPD in some jurisdictions. These practices can be disclosed to patients and can differentiate the service from other skin practitioners. Patients are also usually pleased that their case is being discussed with other physicians. In the setting of a radiation oncology department, the RO is viewed as the leader. It is important that the RO sees this opportunity and takes the initiative. Some qualities of successful skin radiotherapy leaders are summarised in Table 9. Consistent leadership involves constant assessment and reflection on a list of attributes and values. To lead the team effectively, the RO must be proactive and consultative in helping to establish protocols and pathways, especially at the beginning of a new service. Leadership can be tough and lonely.

Table 9 Some qualities of a successful skin RO leader

\begin{tabular}{|c|c|c|}
\hline Number & Relation of quality & Quality \\
\hline $\mathrm{I}$ & Leader themselves & $\begin{array}{l}\text { Consistently behaves according to } \\
\text { agreed core values }\end{array}$ \\
\hline 2 & & Leads by example \\
\hline 3 & & Accepts accountability \\
\hline 4 & & Solves problems in a timely fashion \\
\hline 5 & & $\begin{array}{l}\text { Makes decisions after appropriate } \\
\text { counsel }\end{array}$ \\
\hline 6 & Managing down & Communicates expectations clearly \\
\hline 7 & & Provides feedback constructively \\
\hline 8 & & Recognises and grows talent \\
\hline 9 & & Rewards justly \\
\hline 10 & & $\begin{array}{l}\text { Takes time to teach and to be taught } \\
\text { by the radiation craft groups }\end{array}$ \\
\hline II & & $\begin{array}{l}\text { Creates a culture where the team } \\
\text { feels supported and valued }\end{array}$ \\
\hline 12 & Managing up & Advocates for proper resourcing \\
\hline 13 & & Reports honestly about staff \\
\hline 14 & & $\begin{array}{l}\text { Defends staff from inappropriate } \\
\text { behaviours }\end{array}$ \\
\hline
\end{tabular}

Different radiation craft groups can have particular needs in skin. RTs may need the RO to be present during simulation until good habits of wiring and limb positioning are learnt. The RO and dosimetrist can help each other to create economies and work pathways in skin planning. Nurses may need help in understanding the progressive symptoms, signs and treatment of radiation dermatitis. Knowing how to take comparative photos in good light is also a skill that should be learnt by ROs and nurses. Engaging physics with real patient contact is important. Asking advice from a physicist about appropriate modalities to cover volumes, even in the presence of the patient and family, builds confidence and makes physics staff feel included in the team and on the therapeutic journey. Being introduced to more staff reassures the patient and helps the patient to understand the amount of expertise and people involved in their care.

\section{The skin care medical practitioners including referrers}

To understand where a high-quality radiation skin practice can add value, the skin $\mathrm{RO}$ will benefit from an understanding of the skin care medical community. This overview aims to help the skin RO enter the skin care medical community in a productive and collaborative manner. Current skin care practitioners do a good job in the face of an increasing amount of work and expectation to provide the perfect outcome in terms of functional, cosmetic and oncological outcomes. Many skin conditions are looked after well by primary care physicians known as general practitioners (GPs) in some countries. When the GPs require more back up, the traditional referral pathway has been to a dermatologist. In each cancer sphere, there is usually a cancer surgeon. In skin, this is usually the plastic surgeon. The cancer physician in skin is the dermatologist. Dermatologists are adept at diagnostics like dermoscopy and have non-surgical methods such as topical therapies. The demand for skin checks, at least in Australia, has increased so much that the supply of dermatologists has not kept pace, leading to the creation of the skin cancer GP (SCGPs) to meet demand. These can be accessed directly by the public. There can be tension between the dermatologists and SCGPs over the perceived level of training and scope of practice. Inviting both groups to the same educational meeting may be problematic.

Some dermatologists specialise in Mohs surgery. Mohs surgery is microscopically controlled skin cancer surgery. After each tissue is removed, and while the patient remains anaesthetised, the tissue is prepared and microscopically examined. This is to see if the specimen has been completely excised with negative margins. This examination involves complete circumferential, peripheral and deep margin assessment using frozen section histology (FSH), and this dictates the decision for additional tissue removal. Mohs surgery has been called the "gold standard" treatment when the skin cancer is amenable to surgery. ${ }^{1}$ Yet, there are caveats. These surgeons have not completed a full surgical training program like plastic surgeons, nor a full training program in histopathology, like a dermato-histopathologist. FSH is not as reliable as paraffin sections. Mohs results in a close margin, but is that enough? What is the basis of this being the gold standard? A Cochrane collaboration review from $2007^{31}$ found overall that there has been very little good quality research on treatments for BCC. In this review, surgery and radiotherapy appear to be the most effective treatments with surgery showing the lowest failure rates. Further Cochrane studies in 2009 and again in 2014 for traditional surgery versus Mohs for periorbital BCC found no RCTs. ${ }^{32,33}$ Has enough level one research been done to justify the gold standard title? If RT and Mohs give similar oncological outcomes, will the tissue conservation delivered by RT give better survivorship? The relationship between ROs and Mohs surgeons must be a special focus of collaboration for the skin RO. This relationship needs special help in some countries. ${ }^{34}$ RCTs are needed but will only accrue if all have the required equipoise to have their patients randomised.

All these providers can operate as sole traders, or partnerships, in the community. This demands doctor time and energy in non-clinical areas. The practices are often small and isolated, and this may tend towards a siloed approach to care. Competition can lead to a lack of unity and loss of focus on maximising patient outcomes. Research can be hard to do across different small competing practices. These providers can lack access to the multidisciplinary (MDT) mentality of large acute hospitals. They may not have access to services like dedicated imaging and pathology. They may not have a pathway to other professional groups that are occasionally needed in skin such as the head and neck tumour board and lymphoedema services. An overall approach for the aspiring skin RO is to try to identify what the needs are of the skin care medical community and to try to help them achieve their goals (Table 10). Some needs may include: 
Table 10 Suggested ways in which skin ROs can help the skin care medica community with their needs

\begin{tabular}{ll}
\hline & Suggestions \\
\hline 1 & Solutions for recalcitrant pathology \\
2 & Access to multidisciplinary pathways for complex cases \\
3 & Engage in providing skin surveillance and diagnostics \\
4 & Education especially CPD, authorship \\
5 & Research excellence \\
6 & Promote unity in the community \\
7 & Communication \\
8 & Culture
\end{tabular}

\section{Solutions for recalcitrant pathology}

This is where the skin care physician has little left to offer and is looking for help. One example is VMAT for extended skin field cancerisation (ESFC). ${ }^{2}$ High dose palliative courses $^{3}$ for older folk who are beyond other therapies also helps. Benign conditions such as recurrent keloids can also be treated with immediate post-operative RT.

\section{Access to multidisciplinary pathways for complex cases}

ROs, as generally trained oncologists, are comfortable communicating and chairing with confidence multidisciplinary tumour boards (MDTs) in large acute hospitals. Others in the skin care medical community may not be as comfortable doing this or can lack access to MDTs. The skin RO can set up and chair a skin MDT and motivate the skin community to present and to comment on cases that others present, thus encouraging a culture of inclusivity. Younger specialists are progressively more versed in the importance of MDT care, so starting with them would be a wise choice. This may seem counter intuitive as the older specialist may have a wider referral base. However, the older clinician can find it harder to change treatment and referral patterns. Electronic or virtual MDTs, which Covid has made more acceptable, can include skin care colleagues that are geographically isolated. The skin RO can provide an MDT referral pathway for the primary carer who may feel out of their depth with complex cases. Sometimes they are too busy to attend an MDT, so a skin RO can be their conduit to acute hospital MDTs.

\section{Engage in providing skin surveillance and diagnostics}

Asking the skin medical community to provide skin surveillance and diagnostics for patients who have finished skin RT is wise. The skin RO should also encourage patients to return to the referrer for ongoing skin checks, dermoscopy and biopsies etc. ROs should not be seen to be taking patients away from referrers. On the rare occasion that a patient needs to be observed rather than treated with RT, the skin RO should offer to share the burden of follow up. ${ }^{17}$

\section{Provide education}

The skin RO can help the skin medical community to successfully complete regulatory imposed requirements for continuing professional development (CPD). In Australia, dermatology trainees need to experience an RT department as part of their specialty training. Skin ROs can be proactive in providing this. Trainees helped in this way easily become future referring consultants. Skin care physicians need to know about modern RT and the skin RO has the responsibility to teach. Skin ROs need to emphasise to the skin care medical community that RT has improved. Regular educational meetings at the skin care physicians' clinics, building doctor-to-doctor trust over a meal or coffee, is a big help. Skin ROs can also offer to present at skin conferences or assist referrers with their presentations. Including referrers as co-authors in papers in which their patients have played a role is a great way to collaborate and aids in making them more inquisitive about modern RT. Table 1 shows that this approach does work.

\section{Research excellence}

It is important to base skin RT research on high-quality research platforms via randomised trials and metanalysis etc. In other tumour streams, this is often driven even further by organised patient advocacy which is yet to occur in skin. ROs can bring the rigor of quality research to the skin care community and help build patient advocacy momentum.

\section{Promote unity within the local community}

Skin ROs, especially when networked, can present as a united front. It is best for ROs to regionalise their referral base to the area immediately around their centre rather than to compete for referrals from all over town. Patients needing fractionated treatment want treatment as close as possible to home. By re-referring on to other skin ROs, the community focuses on the patient and access to treatment, rather than making the patient suffer by running them all over town to support a referral network that benefits only the consultant.

\section{Solutions for time-poor referrers}

As an example, the non-skin GP can find it difficult to take a biopsy during a busy clinic. Establishing an in-house biopsy service within the RT department can assist. It is also an opportunity to inform the patient about radiotherapy for the lesion being biopsied. Using a template, ${ }^{15}$ storing and sending a photo to the referrer of the biopsy site, and making a call when the histopathology is returned, builds trust. One can then offer a radiation treatment pathway for the biopsied patient for which the referring doctor is usually grateful.

\section{Communication}

Basic referrer engagement etiquette is necessary especially at the beginning of a referrer relationship. Skin ROs can call a new referrer after an initial consultation with a newly referred patient. The RO can outline the treatment approach and see if the referrer has anything to add. The skin RO may be the only other doctor the referrer interacts with that day, depending on their practice. SCGPs especially like hearing from specialists. They are keen to learn about RT, particularly for patients who are marginal for surgery. They see the results firsthand when patients referred for RT with recalcitrant problems return for follow up, happy and cured.

\section{Culture}

Radiation departments need to have an open, egalitarian and communicative culture in order that all the highly skilled radiation craft groups contribute effectively to patient care. Other skin carer departments may have a more hierarchical organisation that may not promote constructive dialogue. Encouraging visits by clinicians and their staff to the radiation department where each craft group takes responsibility for explaining their own role can help. 


\section{The skin patients}

Skin patients are often different from tradition RT patients. ${ }^{35}$ They have chronic conditions that cause more morbidity than mortality. They can have comorbidities that promote skin cancer (e.g. immunosuppression) or preclude some therapies (e.g. significant coagulation issues that might impede a surgical graft). Some have surgical fatigue and do not wish to lose any more tissue or be hospitalised while a graft (e.g. on the leg) takes. Some are tormented by common chronic skin diseases for which no cause nor has cure been found. ${ }^{7}$ Patients can be ignorant of what radiation treatments are available for skin and currently there is a lack of effective patient advocacy in skin. The time the doctor spends with skin patients and their carers is very important, especially at the initial consultation. Comfortable seating, privacy and eye contact are important in establishing rapport, which are so essential for winning trust for the therapeutic journey. It is important that the room design reflects the quality service. Many tumour sites are best seen through imaging, negating examination, but with skin a thorough examination and inspection of the problem area with a good light is essential. Palpation, besides its clinical use, especially helps to build rapport.

Skin patients can have high expectations. They are frequent visitors to their skin care physicians and may have established relationships over years that are very important and therapeutic to them. Others can have low expectations as they have had conditions for years and are not expecting the skin RO to offer much, despite the enthusiasm of the referrer. These patients often move from being the most sceptical to the most supportive when RT finally solves their problem. They may complain when at the peak of their acute side effects but tend to quickly forget this period as the side effects pass. They may also return for more courses of RT for other areas of skin cancerisation or individual skin lesions.

As time passes, more elderly patients are becoming social media savvy. This increased democratisation of the internet effectively means that patients are self-referring, despite a doctor's name being on the referral. Referrer engagement should be considered to patients and their carers and not just to doctors. Organised skin patient advocacy is in its infancy, mainly because survivors make up the drive behind advocacy. The number of survivors for the killer skin cancers (melanoma and merkel cell carcinoma $(\mathrm{MCC}))^{36}$ is likely to swell as effective drugs are approved. With RT, some patients can be put off by the necessity for fractionated treatment. Some compromise may need to be made when it comes to fractionation e.g. treatment every second day or special fractionation patterns. ${ }^{3}$ Other patients may be inappropriate for RT as they may not be able to lie still during treatment or may be too claustrophobic to handle a mask. Having access in a RO network and to a superficial or kilovoltage skin machine that does not require such a degree of immobilisation is invaluable.

\section{Management}

Management and the doctors have the same motivation at heart - to provide a sustainable high-quality skin RT service. However, if not properly managed, a fault line can develop between these two essential groups. It is important that both groups understand each other and the priorities that drive them (Figure 15). The doctors' prime motivation is to prevent, cure or palliate pathology. Skin ROs are important for providing proper, effective, clinical and ethical direction to a service. In their opinion, there is never enough funding to achieve a high-quality skin service. Management is essential to resourcing a growing skin RT service. Management are particularly interested in sustainability including costs, revenue, and more recently, patient and referrer satisfaction. Skin RT is not renumerated as well as RT in other tumour streams yet can be labour intensive and costly to provide. There is significant capital outlay and not much hope of an early return as the department goes through the learning curve and tries to break into a crowded and competitive skin care market. Management can be put into the unenviable position of having to ration resources. Sometimes they have to say no to the doctors whose billing makes it all possible. Management efficacy can be affected by whether the service is a public or private provider and this needs to be borne in mind by the skin RO group. In a democracy, a public provider eventually answers to a politician who is interested in re-election by voters satisfied with their provision of health services. Public providers may have to deal with an attitude of "work to rule", with waiting lists developing and the department being left behind in terms of innovation. A private service needs to answer to shareholders in being profitable. Both need to understand and respect each other's strengths and weaknesses and finding mutually productive ways of communication.

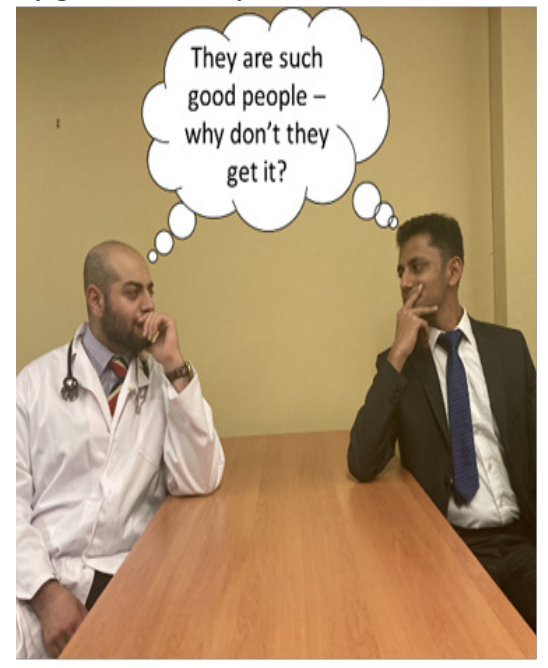

Figure I5 Doctors and management need to understand each other's motives to deliver a sustainable effective skin RT service.

\section{Part 3 The importance of research}

Despite skin cancer being the most common cancer, there is dearth of high-quality evidence to guide radiotherapy treatment. In the country with the highest burden of NMSC, there has only been one Australian RCT involving the radiotherapy of $\mathrm{NMSC}^{37}$ and that trial asked a chemotherapy question. RCTs can easily be done but there is a lack of know-how, leadership and equipoise amongst the skin community to do this. The skin RO can make this happen. Research can be a real bonding experience between the radiation craft groups in the department and can have a positive impact on referrer engagement. Outside the department, medical diplomacy, in a similar fashion to how ROs delivered breast conservation for patients through inclusive research, ${ }^{8}$ can be achieved for skin. The first step, as previously outlined, is to help the skin medical community with their needs through increased RT education in skin and by harnessing patient advocacy. RT education is not helped by the fact that the RT content of most medical courses is very little. For RT education to be effective, the RT medical community needs to create a willing audience by offering an interesting education program through which the fundamentals of RT treatment, such as the need for fractionation and the mechanisms of in-field skin reactions and their treatments, can be clearly explained. The RT staff must take care not to lose the audience with too much RT jargon. Educational meeting sponsorship and patient advocacy can help deliver the funding to achieve this. All 
research involves risk. A risk-averse management-driven research program will not understand how to mitigate risk and will end up requiring levels of approvals that will be inflexible, take too much time to deliver and sap energy and resources. ROs need to drive the research agenda. ${ }^{38}$

\section{Conclusion}

The successful treatment of skin disease decreases morbidity and mortality. Radiotherapy (RT) has a major role as, unlike surgery, RT conserves tissue. Modern RT delivers better conformality and homogeneity, and more is now known about the radiobiology of skin and its diseases, enabling treatment personalisation. Skin needs to be viewed in RT departments as a serious subspecialty especially in Australia. RO leaders are needed to create a niche for RT amongst a crowded field of skin carers. This is achieved by growing a highquality service, understanding the landscape of skin stakeholders, particularly when it comes to appreciating and meeting their needs, and embracing the skin medical community by completing and publishing high-quality research.

\section{Acknowledgments}

The authors wish to thank Aileen Eiszele of A\&L Medical Communications for writing assistance and manuscript preparation. Her work was gratefully supported by Xstrahl. The authors also wish to thank Anthony Tanous for Table 1 (unpublished data), James Hellyer for personal communication about air gaps between bolus and skin, and David Tighe ${ }^{10}$ for Figure 4.

\section{Funding}

None.

\section{Conflicts of interest}

Authors declare that there is no conflict of interest.

\section{References}

1. Wikipedia. Mohs surgery. 1938.

2. Fogarty GB, Christie D, Spelman LJ, et al. Can modern radiotherapy be used for extensive skin field cancerisation: An update on current treatment options. Biomed J Sci \&Tech Res. 2018;4(1).

3. Fogarty GB, McLaren KR, Moutrie Z, et al. Locally advanced skin cancers of the frail and elderly-consider adaptive split course radiotherapy (ASCRT). Br J Dermatol. 2018;179(6):1416-1417.

4. Fogarty GB, Hong A, Scolyer RA, et al. Radiotherapy for lentigo maligna a literature review and recommendations for treatment. $B r J$ Dermatol. 2014;170(1):52-58.

5. Australian Institute of Health and Welfare 2016. Skin cancer in Australia.

6. Jansen MHE, Kessels JPHM, Nelemans PJ, et al. Randomized trial of four treatment approaches for actinic keratosis. $N$ Engl $J$ Med. 2019;380(10):935-946

7. Van Zuuren EJ, Fedorowicz Z, Carter B, et al. Interventions for rosacea Cochrane Database of Systematic Reviews. 2015;4.

8. Darby S, McGale P, Correa C, et al. Early Breast Cancer Trialists' Collaborative Group, Effect of radiotherapy after breast-conserving surgery on 10-year recurrence and 15-year breast cancer death: metaanalysis of individual patient data for 10,801 women in 17 randomised trials. Lancet. 2011;378(9804):1707-1716.

9. Spears P. How advocates help create guidelines. 2017.
10. Tighe DG, Karle B, Hollands A, et al. Definitive radiotherapy for basal cell carcinoma and cutaneous squamous cell carcinoma of the nose. $J$ Dermatol Res. 2019;4(1):156-162.

11. Collins L, Quinn A, Stasko T. Skin cancer and immunosuppression. Dermatol Clin. 2019;37(1):83-94.

12. Fogarty GB, Christie D, Potter A. Volumetric modulated arc therapy for extended skin field cancerisation: Radiobiological learnings from unique patient cases. Int J Radiol Radiat Ther. 2019;6(5):156-162.

13. Skin cancer non melanoma squamous cell carcinoma high risk adjuvant EBRT post operative. 2015.

14. Fogarty G, Muddle R, Sprung C, et al. Unexpectedly severe acute radiotherapy side effects are associated with single nucleotide polymorphisms of the melanocortin-1 receptor. Int J Radiat Oncol Biol Phys. 2009;77(5):1486-492.

15. Alcevski B, Shearer T, Yeong Y, et al. Techniques to verify the correct skin areas for biopsy, treatment, recurrence and in-vivo dosimetry using an A4 plastic sheet as template. Int J Radiol Radiat Ther. 2020;7(4):112-118.

16. Skin Tumours. In: Brierley JD, Gospodarowicz MK, Wittekind C, editors TNM Classification of Malignant Tumours (8th edition). Oxford, UK Wiley-Blackwell; 2017. p. 135-151.

17. Dwyer LJ, Haddad RL, Fogarty GB. Experiences when irradiating grafts and flaps for skin cancer. Int J Radiol Radiat Ther. 2020;7(3):71-76.

18. Fogarty GB, Burt J, Ainslie J. Delay of post operative radiotherapy in high risk skin cancer can be associated with recurrence. $J$ Plast Reconstr Aesthet Surg. 2006;59(2):203-205.

19. Lankshear S, Srigley J, McGowan T, et al. Standardized Synoptic Cancer Pathology Reports-So What and Who Cares?: A Population-Based Satisfaction Survey of 970 Pathologists, Surgeons, and Oncologists. Arch Pathol Lab Med. 2013;137(11):1599-1602.

20. Mullin SJ, Lochhead A, Haddad R, et al. Keratoacanthomas following definitive volumetric modulated arc radiotherapy for skin field cancerization. Int J Radiol Radiat Ther. 2019;6(6):225-232.

21. Prasad S, Dougheney N, Hong A, et al. Scalp hair repigmentation in the penumbral region of radiotherapy- a case series. Int J Radiol Radiat Ther 2020;7(5):151-157.

22. Prescribing, recording, and reporting photon beam therapy (ICRU Report 50). Bethesda: International Commission on Radiation Units and Measurements;1993.

23. Potter A, Price M, Papworth D, et al. A technique for treating extended skin field cancerisation using volumetric modulated arc therapy. Int $J$ Radiol Radiat Ther. 2019;6(4):111-119.

24. Santos DE, Green JA, Bhandari N, et al. Tangential volumetric modulated radiotherapy - A new technique for large scalp lesions with a case study in lentigo maligna. Int J Bioautomation. 2015;19(2):223-236.

25. Arlett $\mathrm{CF}$, Green $\mathrm{MH}$, Rogers $\mathrm{PB}$, et al. Minimal ionizing radiation sensitivity in a large cohort of xeroderma pigmentosum fibroblasts. Brit J Radiol. 2008;81(961):51-58.

26. Butcher LM, Fogarty GB, Sinclair S, et al. Less is more when treating the nasal ala with superficial radiotherapy. Int $J$ Radiol Radiat Ther 2020;7(3):66-69.

27. Fogarty GB, Hong AM, Rocha L, et al. Is more dose and skin reaction required when treating early lentigo maligna definitively with radiotherapy? A case series. Int J Radiol Radiat Ther. 2020;7(5):142-145

28. Fogarty GB, Sullivan J, Gorjiara T et al. Split course superficial radiotherapy in scrotal extramammary paget's disease allows course completion with minimal side effects: A case study. J Dermatol Res. 2020;5(1):202-205 
29. Trotti A, Byhardt R, Stetz J, et al. Common toxicity criteria: version 2.0 . an improved reference for grading the acute effects of cancer treatment impact on radiotherapy. Int J Radiat Oncol Biol Phys. 2000;47(1):13-47.

30. Common Terminology Criteria for Adverse Events (CTCAE). Version 5.0. 2017.

31. Bath-Hextall FJ, Perkins W, Bong J, et al. Interventions for basal cell carcinoma of the skin. Cochrane Database of Systematic Reviews. 2007;1.

32. Narayanan K, Hadid OH, Barnes EA. Mohs micrographic surgery versus surgical excision for periocular basal cell carcinoma. Cochrane Database of Systematic Reviews. 2009;2.

33. Narayanan K, Hadid OH, Barnes EA. Mohs micrographic surgery versus surgical excision for periocular basal cell carcinoma. Cochrane Database Syst Rev. 2014;12.

34. Hruza GJ, Billingsley EM, Brown MD, et al. In regard to the American Society for Radiation Oncology Clinical Practice Guidelines on Radiation Therapy for Basal and Squamous Cell Cancers of the Skin. Pract Radiat Oncol. 2020;10(3):209-210.
35. Fogarty GB, Christie DRH, Kaminski A, et al. A radiation oncology approach for using definitive radiotherapy with volumetric modulated arc therapy (VMAT) for skin field cancerisation (SFC). Int J Radiol Radiat Ther. 2018;5(4):227-234.

36. Amigos. Australasian Merkel Cell Carcinoma Interest Group. 2020.

37. Porceddu SV, Bressel M, Poulsen MG, et al. Postoperative concurrent chemoradiotherapy versus postoperative radiotherapy in high-risk cutaneous squamous cell carcinoma of the head and neck: The randomized phase III TROG 05.01 Trial. J Clin Oncol. 2018;36(13):1275-1283.

38. Gunaratne DA, Veness MJ. Efficacy of hypofractionated radiotherapy in patients with non-melanoma skin cancer: Results of a systematic review. J Med Imaging Radiat Oncol. 2018;62(3):401-411. 